\title{
Influencia de los estereotipos de género en la valoración del incivismo laboral
}

\author{
Isabel Carmona-Cobo ${ }^{1}$, \\ Universidad Católica de Temuco (Temuco, Chile) \\ Eva Garrosa ${ }^{2}$, Bernardo Moreno-Jiménez ${ }^{2}$ y Elena del Barrio ${ }^{2}$ \\ Universidad Autónoma de Madrid (Madrid, España)
}

Este estudio tuvo por objetivo explorar cómo influyen los estereotipos de género en las valoraciones de jóvenes estudiantes sobre las formas de incivismo laboral. Basándonos en la Teoría del Incivismo Selectivo y la Teoría del Rol Social se exploró la existencia de diferencias de género en la detección y tolerancia hacia diferentes formas de incivismo y la percepción de sus consecuencias. Participaron 800 estudiantes españoles (452 mujeres y 348 varones) en un experimento con diseño factorial entre sujetos donde se manipularon las formas de incivismo, el contexto laboral y el género del participante. Los resultados indicaron que mujeres y varones tuvieron valoraciones diferentes. Las mujeres siempre detectaron incivismo, mientras que los varones sólo cuando fue excluyente en lugar de ofensivo, y ambos toleraron menos el incivismo excluyente. Incluso, las mujeres percibieron que el incivismo excluyente reducía la satisfacción laboral en ingeniería y el bienestar emocional en enfermería, mientras que no hubo efectos significativos en varones. En conclusión, el estudio contribuye aportando desarrollos específicos sobre los estereotipos de género, el incivismo laboral, su incidencia y la sensibilización hacia la problemática.

Palabras clave: Estereotipos de género, Incivismo laboral, Estudio experimental.

Influence of gender stereotypes in the rating of workplace incivility

The goal was to explore how gender stereotypes influence young students' ratings of the forms of workplace incivility. Drawing from the theory of selective incivility and the social role theory, the existence of gender differences in the detection and tolerance of different forms of incivility and the perception of its consequences were explored. Participants were 800 Spanish high school and professional training students (452 females and 348 males) in a between-subjects factorial design in which forms of incivility, gender dominated work domain, and participants' gender were manipulated. The results indicated that females and males gave different ratings. Females always detected incivility whereas males only detected it when it was exclusive than offensive, and both genders tolerated exclusive incivility to a lesser extent. In fact, females perceived that exclusive incivility reduced job satisfaction in engineering and emotional well-being in nursing, whereas no significant effects were found in males. To conclude, the study contributes specific developments about gender stereotypes, workplace incivility, its incidence, and sensitization towards the problem.

Keywords: Gender stereotypes, Workplace incivility, Experimental study.

\section{Introducción}

L a literatura sobre las manifestaciones sutiles, no físicas y que implican un trato despectivo interpersonal en el trabajo, ha generado un incremento en los estudios recientes sobre la psicología social aplicada al trabajo (Cortina, 2008; Cortina, Kabat-Farr, Leskinen, Huerta \& Magley, 2011; Cortina, Magley, Williams \& Langhout, 2001; Hershcovis, 2011; Hershcovis \& Reich, 2013). Actualmente, las investigaciones se han centrado en explorar las conductas del sexismo y racismo que pueden actuar como formas "modernas" de discriminación laboral (Cortina et al., 2011; Kabat-Farr \& Cortina, 2012), haciendo referencia a las dinámicas laborales que tienen como resultado la desigualdad entre las trabajadoras y los trabajadores. Los estudios de género, por ejemplo, señalan formas que incluyen el sexismo moderno (Swim, Mallett \& Stangor, 2004), el sexismo sutil (Benokraitis, 1997), el neosexismo (Tougas, Brown, Beaton \& St-Pierre, 1999) y el

1 Facultad de Ciencias Sociales. Carrera de Psicología. Chile.

2 Facultad de Psicología. Departamento de Psicología Biológica y de la Salud. España. 
sexismo contemporáneo (Jackson, Esses \& Burris, 2001). En este sentido, los estereotipos de género pueden estar en el origen de tales actos de discriminación (Betz, Ramsey \& Sekaquaptewa, 2013).

Atendiendo a las características asociadas a cada rol, mujeres y varones ocupan incluso hoy día esferas diferentes en la sociedad, hecho que implica una organización del trabajo diferenciada en base al género (Eagly, Wood \& Diekman, 2000; Wood \& Eagly, 2002). Tradicionalmente, el varón ha ocupado puestos de poder vinculados al ámbito público, mientras que la mujer ha desarrollado labores de cuidado asociadas al ámbito privado (Diekman \& Eagly, 2000; López-Zafra, GarciaRetamero \& Eagly, 2009). Sin embargo, el desarrollo socio-económico de la sociedad ha avanzado a lo largo de los últimos años, dando lugar a un cambio importante en la población trabajadora. Así, las mujeres han pasado a formar parte del mercado laboral y están presentes prácticamente en todos los puestos de trabajo a todos los niveles (Moreno-Jiménez \& Rodríguez-Carvajal, 2013).

No obstante, son las mujeres las que se encuentran en mayor situación de riesgo laboral, sobre todo mujeres jóvenes que desempeñan trabajos poco cualificados. La VII Encuesta Nacional de Condiciones de Trabajo en España (INSHT, 2011) señala que hay un mayor porcentaje de mujeres (8\%) que de varones $(6,7 \%)$ víctimas de agresiones verbales, rumores o aislamiento social. Además, las mujeres $(1,2 \%)$ en mayor medida que los varones $(0,1 \%)$ indican ser víctimas de discriminación sexual o discriminación por razón de género. Recientes estudios apoyan estos resultados y señalan el incivismo laboral como una forma "moderna" de discriminación laboral hacia la mujer (Cortina et al., 2011; Kabat-Farr \& Cortina, 2012).

\section{Incivismo laboral y sus formas}

Andersson y Pearson (1999) definieron el incivismo laboral como una "conducta antisocial de baja intensidad -ruda y descortés- con una intencionalidad ambigua de dañar a la víctima y que viola las normas laborales de respeto mutuo" (p. 457). A diferencia de otras formas de agresión, el incivismo tiene intensidad leve o moderada, viola las normas de respeto mutuo, se manifiesta en interacción con otros y sobre todo, no está muy claro si el perpetrador tiene una intención directa de causar un daño a la víctima. Precisamente esta última característica, la intencionalidad ambigua de causar un daño, es lo que convierte al incivismo en una forma de agresión peculiar.

Una intencionalidad ambigua no exime al incivismo de sus consecuencias negativas a corto y largo plazo. Desde su origen las autoras advirtieron de un efecto de espiral a través del cual la persona que inicialmente era perpetradora acaba finalmente siendo víctima de incivismo (Andersson \& Pearson, 1999). Los estudios han encontrado evidencias que apuntan al incivismo como desencadenante de estrés laboral, distracción cognitiva, reducción de la satisfacción laboral y creatividad, y un aumento en el afecto negativo y en los niveles de rotación laboral (Cortina et al., 2001; Garrosa, Carmona-Cobo, Moreno-Jiménez \& Sanz-Vergel, 2015; Lim, Cortina \& Magley, 2008; Schat \& Kelloway, 2005).

En la actualidad, las consecuencias del incivismo tienen repercusiones directas e inmediatas sobre las mujeres trabajadoras. Cortina (2008) propuso la Teoría del Incivismo Selectivo para referirse al incivismo como una forma "moderna" de discriminación por razón de sexo y raza en las organizaciones. El argumento central es que el incivismo no siempre es "general", sino que a veces constituye una expresión encubierta de exclusión social hacia las mujeres y minorías étnicas dentro de la organización. A pesar de los intentos para erradicar la discriminación entre las trabajadoras y los trabajadores, el incivismo puede actuar como una forma latente de disfuncionalidad en muchos contextos dentro de la organización (Kabat-Farr \& Cortina, 2012). Por tanto, estudiar específicamente cómo se expresa esta forma de agresión y qué repercusiones concretas tiene para 
las víctimas y los observadores supone un avance en la comprensión del incivismo como forma de discriminación laboral por género.

El incivismo se manifiesta en la etapa inicial de formas severas de agresión laboral (Andersson \& Pearson, 1999), y como indican recientes investigaciones, las reacciones de las víctimas pueden variar considerablemente dependiendo de sus formas de expresión (Chui \& Dietz, 2014). Las reacciones pueden ser pasivas (por ejemplo, ignorar el incidente) o activas (tales como, deseos de venganza), por lo que la forma de expresión del incivismo se convierte así en un aspecto relevante en la explicación de tales dinámicas laborales agresivas. En consecuencia, los observadores de incivismo podrían ser más o menos permisivos dependiendo de la forma perpetrada.

Analizando detenidamente la escala utilizada para evaluar esta conducta, la Workplace Incivility Scale (Cortina et al., 2001), se pueden observar diferentes formas de expresar este comportamiento incívico, que oscilan desde formas activas hasta formas pasivas. Por ejemplo, el incivismo tiene lugar cuando compañeros(as) o superiores han dudado del juicio de un(a) trabajador(a) sobre un asunto del que es responsable, o cuando han hecho comentarios humillantes o degradantes sobre el/la trabajador(a). Siguiendo estos ejemplos podríamos pensar que el incivismo se manifiesta mediante conductas activas en las cuales el perpetrador realiza un hecho concreto, ya sea dudar de la responsabilidad de alguien o hacer comentarios humillantes, así se trataría de conductas ofensivas. Sin embargo, también se produce incivismo cuando un(a) trabajador(a) ha sido ignorado(a) o excluido(a) del resto de compañeros(as) o grupo profesional, o cuando han prestado poca atención a sus declaraciones o han mostrado poco interés en su valoración. En este último caso el perpetrador omite a la víctima de incivismo, ya sea ignorándola o prestando poca atención a sus declaraciones, se trataría de conductas excluyentes en las cuales el incivismo se expresa de forma pasiva.

En todos los casos la persona es víctima de incivismo, pero iexisten diferencias entre estas manifestaciones? ¿cómo influyen estas formas de expresión del incivismo en los observadores? Deteniéndonos en los ejemplos mencionados y acorde con la Teoría del Incivismo Selectivo (Cortina, 2008) y las investigaciones actuales (Chui \& Dietz, 2014) planteamos la idea de que el incivismo puede expresarse de diferentes formas. Además, estas formas diversas de expresión podrían estar relacionadas con las reacciones de los observadores de incivismo, encontrándose diferencias de género en sus valoraciones. En base a esta idea, planteamos la existencia de dos formas de incivismo laboral: incivismo ofensivo e incivismo excluyente, que pueden tener consecuencias diversas.

\section{Contexto laboral y género}

La división del trabajo entre mujeres y varones está basada en los roles de género marcados por la sociedad. La Teoría del Rol Social (Eagly, 1987) puede aplicarse al ámbito del trabajo. La teoría asume que la diferencia entre mujeres y varones genera creencias acerca de las características que definen a mujeres y varones, desencadenando una serie de prescripciones sobre los comportamientos asociados a cada rol. Así, se espera que las mujeres sean más sensibles, afectuosas o cuidadosas - de acuerdo con las características típicas del rol femenino - mientras que los varones serán más agresivos, decididos o valientes - características típicamente masculinas.

La división del trabajo en base al sexo tiene su origen en la idea tradicional de que un trabajo remunerado y cualificado es un dominio de varones. Contraria a esta idea conservadora, Kiesler (1975) propuso un punto de vista alternativo, el trabajo llega a ser típico del sexo femenino o masculino de acuerdo con el sexo de la persona que comúnmente lo ha desempeñado. Por tanto, esta desproporción en la representación de uno de los sexos se apoya en la creencia de que el 
trabajo es apropiado para una persona de ese sexo e inapropiado para la de otro sexo, es decir, el trabajo que desempeña una persona en la ocupación de enfermería sería más apropiado para una mujer al ser un contexto laboral tradicionalmente femenino; mientras que la ocupación dentro de un contexto laboral de ingeniería, típicamente masculino, sería más apropiado para un varón.

El lack-of-fit model (Heilman, 1983) propone que la percepción de incongruencia es un elemento crucial en el proceso de división del trabajo. Las expectativas sobre el desempeño laboral de una persona en un trabajo particular están determinadas por el ajuste entre la percepción de los atributos de la persona y la percepción de los requerimientos del trabajo en términos de habilidades. Por tanto, la percepción de un buen ajuste entre la persona y el trabajo incrementa las expectativas de éxito, mientras que la percepción de un mal ajuste incrementa las de fracaso. Partiendo de este modelo, sería razonable pensar que las mujeres opten por trabajos típicamente femeninos y se espere de ellas una conducta acorde con su rol de género. Los atributos de una mujer, por ejemplo, se podrían ajustar en mayor medida a las habilidades requeridas en el trabajo de enfermería, lo que incrementaría las expectativas de éxito de la mujer en su desempeño laboral dentro del contexto de género predominante. Igualmente, las valoraciones que se hicieran de una mujer trabajadora en un entorno laboral típicamente femenino podrían ser diferentes a las valoraciones que se hicieran cuando la mujer trabajara en un contexto masculino no congruente con su rol.

¿Cómo afectan los estereotipos de género a las valoraciones del incivismo según sus formas de expresión y el contexto laboral? Los estereotipos de género son generalizaciones sobre los atributos de mujeres y varones (Heilman, 2012; López-Zafra \& Garcia-Retamero, 2012). Las características atribuidas a cada rol de género permanecen constantes en diferentes culturas (Williams \& Best, 1990) y contextos laborales (Schein, 2001), y son duraderas en el tiempo (Hentschel, Heilman, \& Peus, 2012). Las creencias de jóvenes estudiantes acerca de los roles de género podrían estar reflejando los estereotipos de género a la hora de valorar cual ha de ser la conducta esperada de una mujer trabajadora y cual la esperada de un varón en idénticas circunstancias. De hecho, la evidencia empírica ha mostrado que los estereotipos de género se activan automáticamente en los observadores, influenciando en su valoración y juicio (Banaji \& Hardin, 1996; Banaji, Hardin, \& Rothman, 1993). Las mujeres estudiantes podrían tener valoraciones diferentes a las de sus compañeros varones sobre las dinámicas de incivismo laboral practicadas en un contexto femenino (por ejemplo, enfermería) o masculino (tales como ingeniería), especialmente en la percepción de las consecuencias para la víctima al haber más víctimas mujeres de incivismo laboral que varones (Cortina et al., 2001; Lim, Cortina \& Magley, 2008).

\section{La investigación}

El objetivo general del estudio fue explorar cómo influyen los estereotipos de género en las valoraciones de jóvenes estudiantes sobre las formas de incivismo laboral. Se aplicó el estudio a jóvenes estudiantes sin experiencia laboral para controlar que las valoraciones estuvieran principalmente influidas por los estereotipos de género, eliminando de este modo la experiencia que puede aportar una persona trabajadora.

En primer lugar se planteó la siguiente pregunta de investigación: (1) ¿Tendrán mujeres y varones estudiantes valoraciones diferentes en la detección y tolerancia al incivismo y, en la percepción de la satisfacción laboral y del bienestar emocional del/a empleado(a)? Existe la necesidad de abordar científicamente las diferencias de género (Ryan \& Branscombe, 2013), y cada vez son más las evidencias encontradas que mantienen la idea de que mujeres y varones valoran y son afectadas(os) de forma diferente ante problemas similares (Garrosa Hernández \& Gálvez Herrer, 2013; INSHT, 2011; Lopez-Zafra \& García-Retamero, 2012; Okimoto \& Heilman, 2012). Partiendo de esta pregunta de investigación, proponemos que: 
(1) Mujeres y varones estudiantes tendrán valoraciones diferentes en la detección y tolerancia al incivismo y, en la percepción de la satisfacción laboral y del bienestar emocional del/a empleado(a).

Por tanto, esperamos encontrar diferencias en las puntuaciones medias de mujeres y varones estudiantes en las medidas de las variables dependientes evaluadas: detección del incivismo, tolerancia al incivismo, percepción de la satisfacción laboral del/a empleado(a), percepción del bienestar emocional del/a empleado(a).

La primera pregunta nos condujo a plantearnos la segunda, asumiendo que existan diferencias: (2) ¿En qué se diferenciarán las valoraciones de mujeres y varones estudiantes en la detección y tolerancia al incivismo y, en la percepción de la satisfacción laboral y del bienestar emocional del/a empleado(a)? Como se ha mencionado anteriormente, la Teoría del Incivismo Selectivo de Cortina (2008) sitúa al incivismo como una forma "moderna" de discriminación laboral con graves consecuencias para las mujeres. Probablemente, el hecho de que las mujeres sufran con mayor frecuencia situaciones de incivismo es lo que las puede convertir en personas más sensibles a su detección en comparación con los varones (Cortina et al., 2001; Lim, Cortina \& Magley, 2008) y menos tolerantes especialmente bajo circunstancias congruentes con su rol de género (Eagly, 1987). Por otra parte, el rol masculino instaurado socialmente para los varones podría hacer que las formas de incivismo ofensivo fueran menos detectadas y más toleradas que el incivismo laboral excluyente, siendo un estereotipo más frecuente en mujeres, sobre todo, cuando el contexto laboral de género es predominantemente masculino. Esta idea nos llevó a proponer que:

(2a) Las mujeres detectarán el incivismo laboral en todos los casos, tanto incivismo ofensivo como incivismo excluyente y en ambos contextos laborales, mientras que serán menos tolerantes cuando el incivismo sea excluyente en lugar de ofensivo y el contexto laboral sea enfermería en lugar de ingeniería.

(2b) Los varones detectarán el incivismo laboral y serán menos tolerantes cuando el incivismo sea excluyente en lugar de ofensivo y el contexto laboral sea ingeniería en lugar de enfermería.

Por tanto, esperamos no encontrar diferencias en las puntuaciones medias de mujeres estudiantes en la detección del incivismo, mientras que si esperamos encontrar diferencias en las puntuaciones medias de las participantes en la tolerancia y, en el caso de los varones estudiantes, además esperamos encontrar diferencias en la puntuaciones medias de la detección y tolerancia al incivismo dependiendo de la forma de incivismo presentada y del contexto laboral en el que tenga lugar el incivismo.

Respecto a la percepción de las consecuencias que el incivismo laboral tiene en las víctimas, el presente estudio investiga la percepción de las y los estudiantes sobre el efecto del incivismo en la satisfacción laboral y el bienestar emocional del/a empleado(a). Apoyándonos en la literatura previa sobre el impacto negativo del incivismo (por ejemplo, Garrosa et al., 2015; Lim, Cortina \& Magley, 2008) y la división del trabajo entre mujeres y varones que dibuja contextos laborales diferenciados por género (Eagly, 1987; Heilman, 1983), proponemos lo siguiente:

(2c) Las mujeres percibirán una reducción de la satisfacción laboral y del bienestar emocional del/a empleado(a) cuando el incivismo sea excluyente en lugar de ofensivo y el contexto laboral sea enfermería en lugar de ingeniería.

(2d) Los varones percibirán una reducción de la satisfacción laboral y del bienestar emocional del/a empleado(a) cuando el incivismo sea excluyente en lugar de ofensivo y el contexto laboral sea ingeniería en lugar de enfermería.

Por tanto, esperamos encontrar diferencias en las puntuaciones medias de mujeres y varones estudiantes en la percepción de la satisfacción laboral y del bienestar emocional del/a empleado(a) dependiendo de la forma de incivismo presentada y del contexto laboral en el que tenga lugar el incivismo. 


\section{Método}

\section{Participantes y procedimiento}

La muestra total se compuso de 800 estudiantes españoles de Educación Secundaria no obligatoria (452 mujeres, 348 varones) con una media de edad de 18 años $(D T=2.29)$ y sin experiencia laboral, el 56.4\% estudiaba Bachillerato y el 43.6\% Formación Profesional. Se contactó con diversos centros de enseñanza y se informó del procedimiento para participar en el estudio. La directiva de los centros informó al profesorado, padres y madres, y al alumnado de la posibilidad de participar en el estudio. Finalmente se seleccionaron las y los participantes que cumplían los criterios de inclusión: alumnas y alumnos de Bachillerato o Formación Profesional sin experiencia laboral. En primer lugar, las mujeres y varones participantes firmaron un consentimiento informado y, a continuación, fueron asignados de forma aleatoria a los grupos experimentales. Completaron las condiciones experimentales en formato electrónico aproximadamente durante 15 minutos. En base a estudios similares, las condiciones experimentales consistían en la presentación de un texto y seguidamente unas preguntas referidas al texto que las y los participantes habían de responder (Okimoto \& Heilman, 2012).

\section{Diseño}

\section{Variables independientes}

Se manipularon las siguientes variables independientes: Formas de incivismo (ofensivo, excluyente); Contexto laboral (enfermería, ingeniería) y Género del participante (mujer, varón). Se trata de un diseño factorial entre sujetos 2 (Formas de incivismo) x 2 (Contexto laboral) x 2 (Género del participante). La Tabla 1 presenta el diseño del estudio y la distribución de mujeres y varones participantes en los grupos experimentales.

Tabla 1. Diseño del estudio y distribución de las y los participantes por grupo experimental

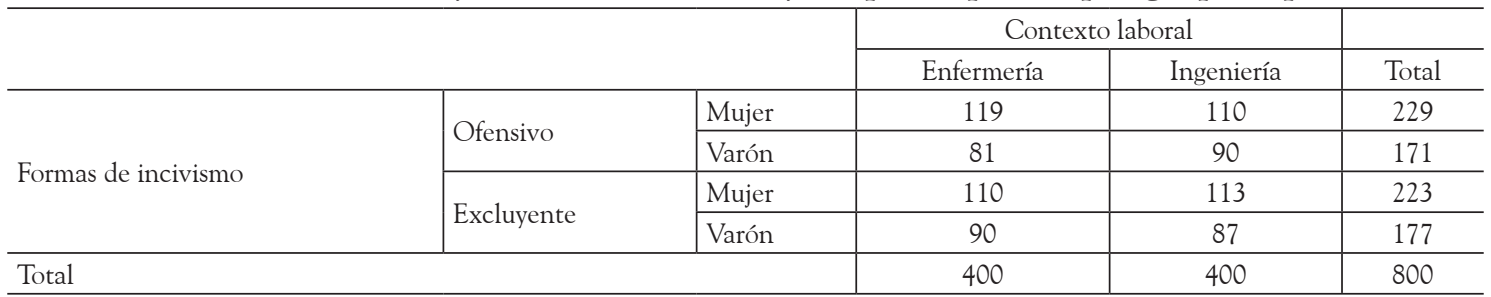

\section{Medición de las variables dependientes}

Se preguntó a las mujeres y varones de la muestra acerca de su valoración sobre el comportamiento del/a jefe(a) y la situación laboral descrita en el texto. En línea con Okimoto y Heilman (2012), el texto de las condiciones experimentales describía una situación de incivismo laboral perpetrada por un(a) jefe(a) hacia un(a) empleado(a), manipulando las variables independientes anteriormente mencionadas. La descripción del texto fue exactamente la misma, excepto la variación reflejada por las variables independientes manipuladas en cada condición experimental. A continuación se presenta la evaluación de las variables dependientes. La Tabla 2 muestra el Coeficiente Alpha y el Coeficiente de Correlación de Pearson que dan cuenta de la fiabilidad de las escalas utilizadas y la interrelación de las variables, respectivamente. 
Variables control. Se registraron las características sociodemográficas con el objeto de controlar la homogeneidad de la muestra y aplicar los criterios de inclusión. Las y los participantes informaron el género, edad, nivel de estudios y experiencia laboral.

Detección del incivismo. En base a la definición de incivismo laboral (Andersson \& Pearson, 1999) se crearon diez adjetivos para describir este comportamiento agresivo. En una escala tipo Likert que oscilaba de 1 ( $\mathrm{Si}$, totalmente de acuerdo) a 4 (No, totalmente en desacuerdo) las y los participantes dieron su valoración respecto al comportamiento del/a jefe(a) descrito previamente en el texto que habían leído. Por ejemplo, las y los participantes respondieron a las siguientes cuestiones: "Según tu valoración, este comportamiento del jefe es: maleducado, grosero, descortés, [...] intencionado para causar un daño al empleado". De modo que expresaban su grado de acuerdo/ desacuerdo entre el adjetivo presentado y el comportamiento del/a jefe(a) descrito previamente en el texto. Así, un(a) participante que puntuara 1 ( $\mathrm{Si}$, totalmente de acuerdo) indicaba que había detectado un comportamiento incívico del/a jefe(a) hacia el/la empleado(a) al estar de acuerdo con las características que definían ese comportamiento de incivismo laboral. Por el contrario, un(a) participante que puntuara 4 ( $\mathrm{No}$, totalmente en desacuerdo) expresaba su discordancia con el adjetivo que describía incivismo, y por tanto, ese comportamiento no era detectado como incivismo.

Tolerancia al incivismo. El grado de tolerancia de las y los participantes hacia el incivismo laboral descrito en el texto fue evaluado usando ocho adjetivos bipolares con una escala de respuesta de 7 puntos: "Según tu valoración, esta situación laboral es o puede ser: tolerable/ intolerable, apropiada/inapropiada, soportable/insoportable, [...] aceptable/inaceptable". Siguiendo este ejemplo, puntuaciones próximas a 1 indicaban que el/la participante valoraba que la situación laboral era o podía ser tolerable, mientras que puntuar 7 indicaba una situación laboral intolerable. Se incluyeron dos ítems inversos, "mejorable/inmejorable" y "corregible/incorregible" con el fin de evitar el sesgo de respuesta.

Percepción de la satisfacción laboral del/a empleado(a). Las mujeres y los varones participantes expresaron su valoración respondiendo a un ítem dicotómico para expresar la percepción de las consecuencias del incivismo en el nivel de satisfacción laboral del/a empleado(a): "Según tu valoración, esta situación laboral: Aumenta la satisfacción laboral del empleado/Reduce la satisfacción laboral del empleado". Puntuar con un 1 en la escala de respuesta bipolar indicaba que el/la participante percibía que el incivismo presentado aumentaba la satisfacción laboral del/a empleado(a). Por el contrario, puntuar con un 2 significaba que el/la participante percibía que el incivismo presentado reducía la satisfacción laboral del/a empleado(a). Al tratarse de un único ítem dicotómico no se pudo hallar el Coeficiente Alpha porque la fiabilidad alude a la escala total, y no al ítem (Abad, Olea, Ponsoda \& García, 2011) (ver Tabla 2). Un objetivo concreto del estudio es conocer la valoración de las y los estudiantes sobre las consecuencias del incivismo en la satisfacción laboral de la víctima, por tanto, al igual que otros estudios se optó por preguntar directamente esta variable con un ítem (Beattie \& Griffin, 2014; Okimoto \& Heilman, 2012; Ramsey, Betz \& Sekaquaptewa, 2013).

Percepción del bienestar emocional del/a empleado(a). Con el fin de conocer la percepción de las y los participantes sobre las consecuencias en el bienestar emocional del/a empleado(a), se añadió la Affect Balance Scale (Bradburn, 1969) adaptada al estudio. La escala se compone de diez ítems que evalúan el equilibrio entre los estados emocionales positivos y negativos. Para obtener las puntuaciones de la escala, a cada respuesta afirmativa en la escala de afecto positivo se le asigna un punto y eso también ocurre con la escala de afecto negativo. La puntuación total en la escala es el resultado de restar el afecto negativo del afecto positivo y sumar la constante cinco (para eliminar los valores negativos). De este modo se obtiene una puntuación en un rango que va desde 1 (equilibrio afectivo más bajo) hasta 9 (equilibrio afectivo más alto). Las y los participantes señalaron de 1 ( $\mathrm{Si}$, totalmente de acuerdo) a 4 ( $\mathrm{No}$, totalmente en desacuerdo): "Según tu valoración, a causa de esta situación laboral el empleado se sentirá en su trabajo: Especialmente 
interesado, tan inquieto que no podrá estar sentado por mucho tiempo en su puesto de trabajo, orgulloso porque su jefe le felicitó por algo que hizo, [...] depresivo o muy infeliz por esta situación laboral”. Por tanto, puntuaciones próximas a 1 significaban que el/la participante percibía que el incivismo laboral reducía el bienestar emocional y las próximas a 9 indicaban que se percibía un aumento del bienestar emocional del/a empleado(a).

Tabla 2. Fiabilidad y correlaciones de las medidas de las variables dependientes para mujeres y varones

\begin{tabular}{|c|c|c|c|c|c|c|}
\hline & $\alpha$ Mujeres & $\alpha$ Varones & 1 & 2 & 3 & 4 \\
\hline 1. Detección & .84 & .89 & & $-.48 * *$ & $-.28 * *$ & $-.35 * *$ \\
\hline 2. Tolerancia & .79 & .83 & $-.42 * *$ & & $.32 * *$ & $.38 * *$ \\
\hline 3. Satisfacción laboral & - & - & $-.24 * *$ & $.33 * *$ & & $.41 * *$ \\
\hline 4. Bienestar emocional & .78 & .79 & $-.36 * *$ & $.29 * *$ & $.30 * *$ & \\
\hline
\end{tabular}

Nota: Las correlaciones para las mujeres se presentan en la parte inferior de la diagonal, para los varones en la parte superior $* p<.05, * * p<.01, * * * p<.001$

\section{Resultados}

Se realizó un ANOVA de un factor para cada variable dependiente con el fin de dar respuesta a la primera pregunta de investigación y, de este modo, (1) explorar si mujeres y varones estudiantes tenían valoraciones diferentes en la detección y tolerancia al incivismo, y en la percepción de la satisfacción laboral y del bienestar emocional del/a empleado(a). Los análisis de varianza mostraron un efecto significativo en la detección del incivismo, $F(1,799)=11.99, p<.001$, la tolerancia al incivismo, $F(1,799)=23.07$, $p<.001$, y la percepción del bienestar emocional del/a empleado(a), $F(1,799)=6.55, p<.01$. No se encontraron resultados significativos en la percepción de la satisfacción laboral del/a empleado(a), $F(1,799)=2.27, p=.13$. Por tanto, tal y como planteábamos (1) mujeres y varones estudiantes tuvieron valoraciones diferentes en la detección y tolerancia al incivismo y en la percepción del bienestar emocional del/a empleado(a). Los resultados indicaron diferencias de género en las valoraciones de las variables dependientes evaluadas, excepto en la percepción de la satisfacción laboral.

Con el fin de responder la segunda pregunta de investigación y (2) conocer en qué se diferenciaron las valoraciones de mujeres y varones estudiantes en la detección y tolerancia al incivismo y, en la percepción de la satisfacción laboral y del bienestar emocional del/a empleado(a) se realizaron ANOVAs 2 (Formas de incivismo) x 2 (Contexto laboral) x 2 (Género del participante) para cada una de las mediciones de las variables dependientes (ver Tabla 3).

Tabla 3. Medias y desviaciones típicas de mujeres y varones dependiendo de las formas de incivismo y del contexto laboral

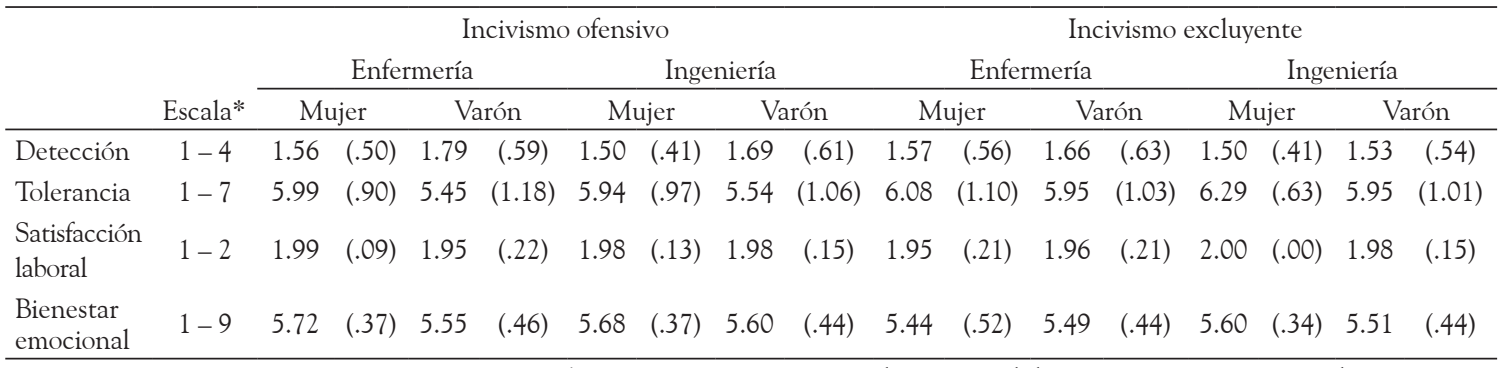

Nota: Desviaciones Típicas entre paréntesis. * Detección: 1 = mayor detección del incivismo, $4=$ menor detección del incivismo. Tolerancia: 1 = mayor tolerancia al incivismo, 7 = menor tolerancia al incivismo. Satisfacción laboral: 1 = aumenta la satisfacción laboral; 2 = reduce la satisfacción laboral. Bienestar emocional: Escala de respuesta tipo Likert 1 - 4 transformada en 1 - 9 al aplicar la corrección de la Affect Balance Scale (Bradburn, 1968), 1 = reduce el bienestar emocional, 9 = aumenta el bienestar emocional 


\section{Detección del incivismo}

El análisis de varianza realizado para la variable dependiente detección del incivismo indicó un efecto principal de las formas de incivismo en los varones, $F(1,344)=5.13, p<.05, \eta^{2}=$ .02 , y no hubo efecto significativo del contexto laboral, $F(1,344)=3.03, p=.08$. En las mujeres no hubo efecto significativo de las formas de incivismo, $F(1,448)=.00, p=.98$, ni del contexto laboral, $F(1,448)=2.22, p=.14$. Estos resultados significan que las mujeres siempre detectaron el incivismo laboral independiente de la forma de incivismo y del contexto laboral, mientras que los varones sólo detectaron el incivismo excluyente, independiente del contexto laboral. En línea con lo que esperábamos encontrar, (2a) las mujeres detectaron el incivismo laboral en todos los casos, tanto incivismo ofensivo como incivismo excluyente y en ambos contextos laborales, mientras que (2b) los varones detectaron el incivismo laboral cuando fue excluyente en lugar de ofensivo y en ambos contextos laborales, no solo en ingeniería.

\section{Tolerancia al incivismo}

Los resultados del análisis de varianza para la tolerancia al incivismo como variable dependiente indicaron un efecto principal de las formas de incivismo en mujeres, $F(1,448)=6.48, p<.05, \eta^{2}=$ .01 , y en varones, $F(1,344)=15.64, p<.001, \eta^{2}=.04$. Sin embargo, no hubo efectos significativos del contexto laboral en mujeres, $F(1,448)=.89, p=.35$, ni en varones, $F(1,344)=.14, p=.71$. Esto significa que tanto las mujeres como los varones mostraron menor tolerancia al incivismo cuando fue excluyente, independiente del contexto laboral en que se presente. Por tanto, en base a los resultados podemos mantener la propuesta de que (2a) y (2b) mujeres y varones fueron menos tolerantes cuando el incivismo fue excluyente en lugar de ofensivo. Por el contrario, mujeres y varones fueron menos tolerantes al incivismo excluyente en ambos contextos laborales, tanto en enfermería como en ingeniería, y no solo en el contexto laboral congruente con el rol de género.

\section{Percepción de la satisfacción laboral del/a empleado(a)}

Se realizó un análisis de varianza para analizar la percepción de las y los participantes respecto a las consecuencias del incivismo en el nivel de satisfacción laboral del/a empleado(a). Los resultados indicaron una interacción significativa entre las formas de incivismo y el contexto laboral en mujeres, $F(1,448)=4.99, p<.05, \eta^{2}=.00$, no obstante no se encontraron efectos significativos en varones, $F(1,344)=.21, p=.86$. Estos resultados significan que cuando se presentó la situación laboral en la que un(a) jefe(a) perpetraba incivismo excluyente hacia un(a) empleado(a), las mujeres percibieron una reducción de la satisfacción laboral del/a empleado(a) cuando esta situación laboral tenía lugar en el contexto de ingeniería en comparación con el de enfermería, $F(1,792)=4.74, p<.05, \eta^{2}$ $=.00$, (ver Gráfico 1). Por tanto, en contra de lo que esperábamos los resultados señalaron que (2c) las mujeres percibieron una reducción de la satisfacción laboral cuando el contexto laboral fue ingeniería en lugar de enfermería. Sin embargo y a favor de lo planteado, esta percepción se produjo cuando el incivismo fue excluyente. Respecto a los varones, (2d) ellos no mostraron diferencias en la percepción de la satisfacción laboral del/a empleado(a), en contra de nuestras expectativas. Por lo tanto, independiente de la forma de incivismo presentada y del tipo de contexto en el que apareció, todos los varones mostraron las mismas valoraciones. 


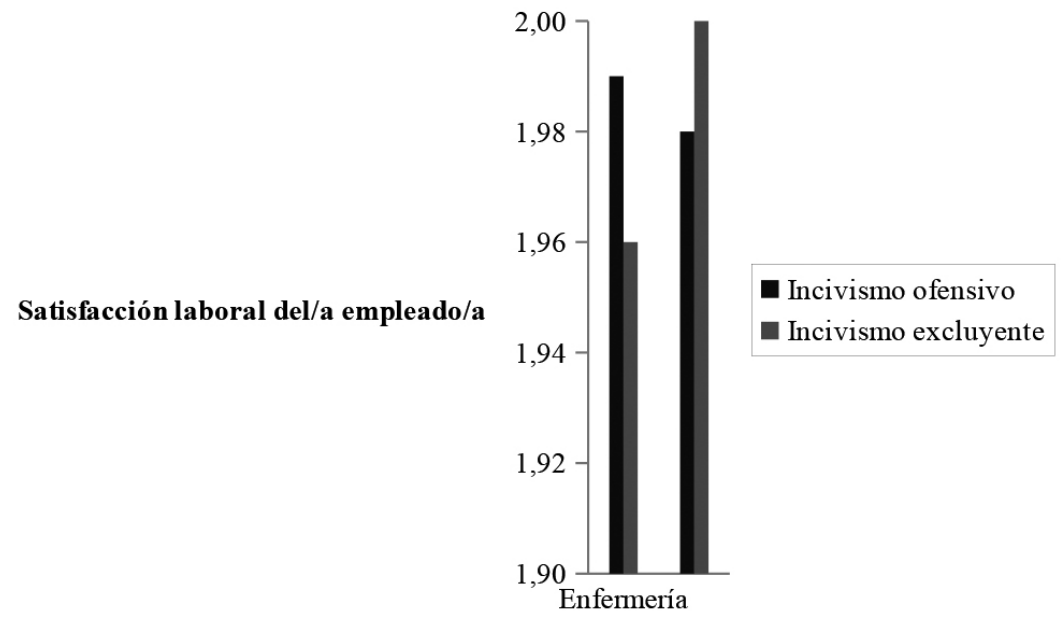

Gráfico 1. Percepción de las mujeres sobre la satisfacción laboral del/a empleado(a) Nota: Escala de respuesta: 1 = aumenta la satisfacción laboral; 2 = reduce la satisfacción laboral

\section{Percepción del bienestar emocional del/a empleado(a)}

Se realizó un análisis de varianza para analizar la percepción de las y los participantes sobre las consecuencias del incivismo en el bienestar emocional del/a empleado(a). De nuevo, los resultados mostraron una interacción significativa entre las formas de incivismo y el contexto laboral en mujeres, $F(1,448)=6.43, p<.05, \eta^{2}=.01$; mientras que no se encontraron efectos significativos en varones, $F(1,344)=.09, p=.77$. Acorde con nuestro planteamiento, (2c) cuando se presentó la situación laboral en la que un(a) jefe(a) perpetraba incivismo excluyente hacia un(a) empleado(a), las mujeres percibieron una reducción del bienestar emocional del/a empleado(a) cuando esta situación laboral tenía lugar en el contexto de enfermería en comparación con el de ingeniería, $F(1,792)=7.96, p<.01, \eta^{2}=.01$. Además, cuando la situación de incivismo se presentó en el contexto de enfermería, las mujeres percibieron una reducción del bienestar emocional del/a empleado(a) cuando el incivismo fue excluyente en lugar de ofensivo, $F(1$, $792)=23.99, p<.001, \eta^{2}=.03$, (ver Gráfico 2). Sin embargo, $(2 \mathrm{~d})$ los varones no mostraron diferencias significativas en la percepción del bienestar emocional del/a empleado(a) por lo que, independiente de la forma de incivismo presentada y del tipo de contexto en el que apareció, todos los varones mostraron las mismas valoraciones.

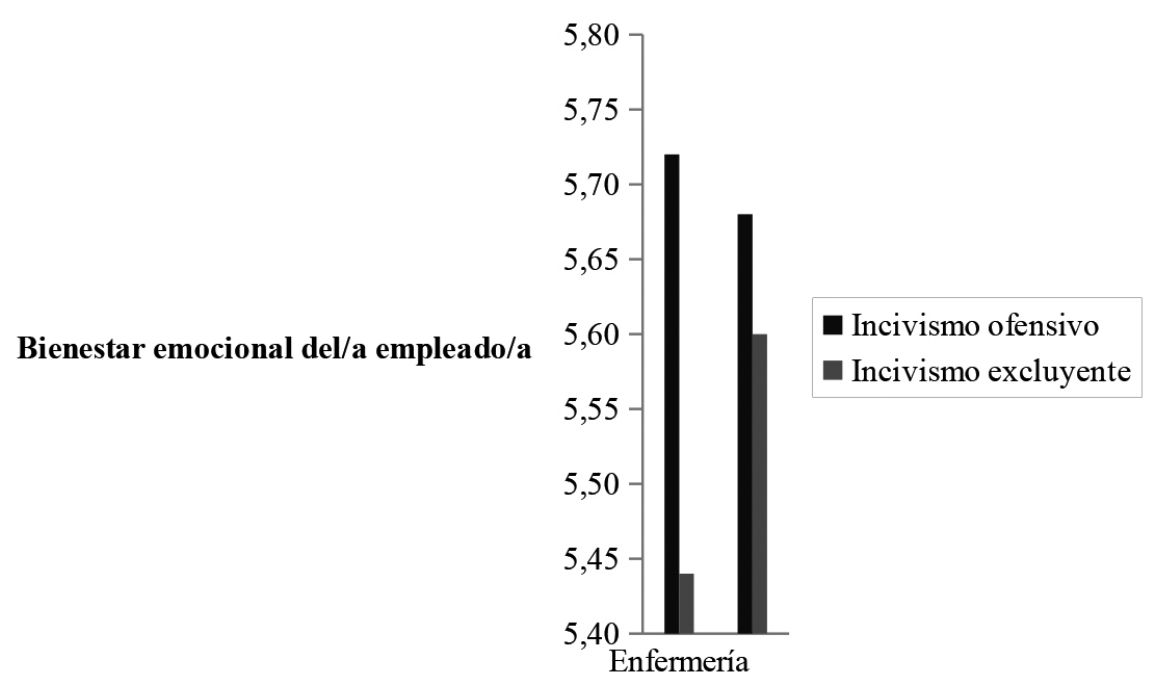

Gráfico 2. Percepción de las mujeres sobre el bienestar emocional del/a empleado(a)

Nota: Escala de respuesta tipo Likert 1 - 4 transformada en 1 - 9 al aplicar la corrección de la Affect Balance Scale (Bradburn, 1968), 1 = reduce el bienestar emocional, 9 = aumenta el bienestar emocional 


\section{Discusión}

El objetivo general del presente estudio fue explorar cómo influyen los estereotipos de género en las valoraciones de jóvenes estudiantes sobre las formas de incivismo laboral. Actualmente, el incivismo representa una forma "moderna" de discriminación en el trabajo, y su estudio desde la perspectiva de género se ha convertido en un tema central en las investigaciones (Cortina et al., 2011; Hershcovis, 2011; Hershcovis \& Reich, 2013). Los resultados de este estudio indican que las mujeres detectan el incivismo en todos los casos, mientras que los varones sólo lo hacen cuando es excluyente en lugar de ofensivo, y ambos toleran menos el incivismo excluyente. Incluso, las mujeres perciben que el incivismo excluyente reduce la satisfacción laboral en ingeniería y el bienestar emocional en enfermería, mientras que no hay diferencias significativas en varones.

Estos resultados destacan la influencia de los estereotipos de género señalando diferencias en las valoraciones entre mujeres y varones estudiantes sobre dinámicas laborales agresivas en la organización. Recientemente, el incivismo laboral es una de las formas de agresión que más interés ha suscitado dentro del marco de la salud laboral (Chui \& Dietz, 2014; Cortina et al., 2011; Hershcovis, 2011; Hershcovis \& Reich, 2013; Schat \& Kelloway, 2005). Comprender los aspectos relacionados con esta problemática laboral requiere una aproximación metodológica adecuada. Acorde con la literatura, el estudio experimental realizado ha permitido comprobar cómo los estereotipos de género pueden actuar de forma automática influyendo en las percepciones de estudiantes expuestos a ejemplos de diferentes formas de incivismo laboral en distintos contextos laborales asociados al género (Banaji \& Hardin, 1996; Banaji, Hardin \& Rothman, 1993). Además, las diferencias de género encontradas subrayan la importancia de analizar estas percepciones por separado en mujeres y varones. Los resultados del estudio muestran la existencia de variables de género que estarían influenciando en los observadores de incivismo. Concretamente no todas las personas muestran el mismo grado de permisividad hacia estas conductas, y son las mujeres las menos permisivas tanto en su detección como en la percepción de sus consecuencias. No obstante, las diferencias de género encontradas podrían deberse a otras variables como las diferencias individuales o la historia personal de cada persona.

Las evidencias encontradas en el presente estudio ponen de manifiesto que mujeres y varones estudiantes tienen valoraciones diferentes ante situaciones laborales idénticas. Los resultados justifican el estudio de la agresión laboral analizando la influencia de los estereotipos de género en mujeres y varones, tal y como plantean otras investigadoras (Betz et al., 2013; Ryan \& Branscombe, 2013), especialmente cuando el incivismo laboral es más frecuente en mujeres que en varones (Cortina et al., 2001; Lim, Cortina \& Magley, 2008). Probablemente esta diferencia entre las valoraciones de mujeres y varones estudiantes puede deberse a la diferenciación social que establecen los roles de género y las creencias de los estudiantes acerca de los estereotipos de género (Heilman, 2012; Heilman \& Eagly, 2008; López-Zafra \& Garcia-Retamero, 2012). Investigar estas formas de incivismo en jóvenes sin experiencia laboral radica en la importancia de adoptar medidas preventivas, para ello, la Agencia Europea para la Seguridad y Salud en el Trabajo (2006) señala como primer paso la identificación de riesgos. La presente investigación ha permitido identificar el incivismo como factor latente de discriminación laboral por género de modo concreto en mujeres y varones estudiantes observadores de sus distintas formas de expresión. Los resultados ponen de manifiesto la necesidad de aplicar intervenciones psicoeducativas que fomenten la educación en igualdad en edades clave para el desarrollo de creencias estereotipadas con el fin de que las formas indirectas y sutiles de agresión no pasen inadvertidas.

El estudio plantea la existencia de dos formas de incivismo laboral, incivismo ofensivo e incivismo excluyente. Esta investigación avanza también en esta línea de distinción y sus resultados resaltan la importancia de analizar el incivismo de forma particular y específica. Partiendo de la 
escala de incivismo laboral (Cortina et al., 2001), la Teoría del Incivismo Selectivo (Cortina, 2008) y las investigaciones actuales sobre los observadores de incivismo (Chui \& Dietz, 2014), los efectos principales encontrados en las formas de incivismo podrían estar constatando la existencia de estas dos formas de agresión. En este sentido, el incivismo puede manifestarse de diferentes modos y al no actuar como una forma de separación general puede representar una forma encubierta de exclusión social hacia las mujeres en la organización y desencadenar diferentes reacciones en las víctimas y personas que observan sus distintas formas de expresión. Este hallazgo dirige las intervenciones psicosociales concretamente hacia aquellas formas pasivas de incivismo que pueden pasar desapercibidas y que, sin embargo, desencadenan un malestar en la víctima.

¿En qué se diferencian las valoraciones de mujeres y varones estudiantes en la detección y tolerancia al incivismo? En todos los casos las mujeres detectan el incivismo laboral, sea cual sea la forma y el contexto en que se manifieste, mientras que los varones solo lo detectan cuando el incivismo es excluyente. En línea con las investigaciones previas, los resultados nos llevan a mantener la idea inicial de que las mujeres serían más sensibles que los varones a las situaciones de incivismo laboral (Cortina, 2008; Cortina et al., 2001; Lim, Cortina \& Magley, 2008), en gran medida, porque son las más discriminadas por este tipo de agresión (Cortina et al., 2011). Incluso, acorde con nuestro planteamiento, mujeres y varones toleran menos el incivismo excluyente. Conforme a los roles de género esperados (Eagly, 1987), sólo cuando los varones se sienten excluidos o menospreciados (el caso del incivismo excluyente) reaccionan mostrando su intolerancia hacia esta situación laboral, mientras que las formas ofensivas de incivismo características pasarían más inadvertidas. Puede que los varones detecten y muestren menos tolerancia al incivismo sólo cuando es excluyente porque iría en contra de su rol de género. En este sentido, la exclusión puede resultarles más agresiva ya que implicaría apartarles de los asuntos relacionados con el trabajo. Desde este punto de vista es importante la psicoeducación, ya que cuando no se detecta una conducta incívica puede aumentar su tolerancia y frecuencia.

¿En qué se diferencian las valoraciones de mujeres y varones estudiantes en la percepción de la satisfacción laboral y del bienestar emocional de la víctima de incivismo? Un aporte destacado en la investigación es conocer qué consecuencias del incivismo laboral perciben las personas para la víctima. Las mujeres valoran que el incivismo excluyente reduce la satisfacción laboral en el contexto de ingeniería, es decir, dan más importancia a las consecuencias del incivismo cuando la víctima trabaja en un contexto típicamente masculino. Incluso las mujeres perciben peores consecuencias cuando el entorno es típicamente masculino, resultado que se esperaría en los varones, quienes por el contrario no perciben consecuencias significativas. Este resultado apoyaría la idea de que incluso hoy día las mujeres jóvenes mantienen creencias estereotipadas sobre las expectativas del desempeño laboral en contextos asociados al género, valorando más negativamente las repercusiones del incivismo en un contexto tradicionalmente masculino.

Respecto a la percepción de las consecuencias del incivismo en el bienestar emocional de la víctima, las mujeres perciben que el incivismo excluyente reduce el bienestar emocional de la víctima que trabaja en enfermería. Por el contrario, los varones no perciben consecuencias emocionales significativas. Estos resultados apoyan las evidencias previas sobre las consecuencias negativas que puede tener el incivismo laboral en el bienestar emocional de las mujeres (Garrosa et al., 2015; Lim, Cortina \& Magley, 2008) y ponen de relieve la necesidad de estudiar las consecuencias por separado en mujeres y varones (Ryan \& Branscombe, 2013), así como el escaso conocimiento de las consecuencias en estas formas de relación psicosocial.

Si bien la detección y tolerancia están determinadas únicamente por la forma de incivismo presentada, en la percepción de las consecuencias entran en juego otros factores fundamentales como es el contexto laboral del género predominante. Estos resultados corroboran las creencias estereotipadas de los jóvenes sobre el rol que ha de desempeñar una mujer trabajadora y el rol que 
debe desempeñar un varón trabajador (Eagly et al., 2000; Wood \& Eagly, 2002) y la influencia del género en la división del trabajo (Eagly, 1987; Heilman, 1983; Kiesler, 1975). De modo que ante la misma situación de incivismo laboral, el hecho de que el contexto laboral sea masculino o femenino puede cambiar la valoración de las personas y se puede percibir mayor intensidad de consecuencias.

El estudio, que contribuye a la investigación sobre la importancia de las características específicas de las conductas incívicas y reafirma las problemáticas laborales existentes, no está exento de limitaciones. Por ejemplo, la investigación carece de un grupo control que presente el incivismo en un contexto no vinculado al género. Un aspecto importante que no se contempla en este trabajo es el papel que pueden desempeñar las diferencias de sexo del perpetrador y de la víctima. Esto es, a las variables independientes estudiadas habría que añadir la influencia del sexo del/a supervisor(a) y el sexo del/a empleado(a) para conocer su efecto en las variables dependientes evaluadas. Por otra parte, sería interesante aplicar el estudio a personas trabajadoras víctimas de incivismo laboral, sólo de este modo podemos conocer el efecto real - y no la percepción - del incivismo laboral, así como observar las diferencias entre las distintas formas de incivismo planteadas en este estudio. También, incluir medidas para evaluar las creencias estereotipadas de las mujeres y varones participantes aportaría un mayor control, especialmente cuando se trata de conocer la influencia de los estereotipos de género. En línea con investigaciones similares, previo a la aplicación de las condiciones experimentales, se deberían evaluar las creencias sobre los roles de género mediante la recopilación de sus adjetivos característicos (Okimoto \& Heilman, 2012). Esta medida resultaría útil para el análisis y la interpretación de los resultados. Las mediciones de las variables dependientes se podrían mejorar, por ejemplo, utilizando instrumentos de evaluación estandarizados, sobre todo sería ventajoso para ampliar la evaluación de la satisfacción laboral, que sólo se evalúa con una pregunta. No obstante, también otros estudios utilizan medidas de un único ítem cuando es pertinente al objeto de estudio (Beattie \& Griffin, 2014; Okimoto \& Heilman, 2012; Ramsey, Betz, \& Sekaquaptewa, 2013). Cabría tener en cuenta estas limitaciones para futuras investigaciones relacionadas con esta temática.

La investigación realizada añade aportaciones relevantes al estudio de la agresión laboral desde la perspectiva de género. Se trata de un diseño experimental a través del cual podemos establecer relaciones de causa y efecto, hasta ahora poco aplicado en los estudios sobre agresión laboral (Hershcovis \& Reich, 2013). Se estudian las valoraciones de jóvenes que todavía no se han incorporado al mercado de trabajo, por lo que es importante conocer el efecto de los estereotipos de género a este nivel para implementar medidas de prevención y promover la educación en igualdad. Una aportación destacada es la propuesta de la existencia de diferentes formas de incivismo laboral que, como hemos comprobado, pueden tener consecuencias diversas. Finalmente, las variables dependientes estudiadas pueden informarnos de los procesos que mantienen las dinámicas laborales incívicas y conocer sus mecanismos desde la influencia de los estereotipos de género.

\section{Conclusiones}

Recientes estudios nos han permitido conocer los efectos negativos del incivismo laboral de forma diaria (Garrosa et al., 2015) y experimental (Porath \& Pearson, 2010). El incivismo representa una forma de discriminar a las mujeres en el ámbito laboral (Cortina, 2008; Cortina et al., 2011; Kabat-Farr \& Cortina, 2012) y supone un problema a nivel personal, organizacional y social dadas las consecuencias derivadas de esta dinámica tóxica de trabajo. La realización de este estudio ha permitido conocer cómo influyen los estereotipos de género en las valoraciones de jóvenes estudiantes que próximamente se incorporarán al mercado laboral. Intervenir en esta etapa es imprescindible para detectar creencias que puedan desencadenar futuros actos de discriminación, así como las características del comportamiento incívico y sus consecuencias. 
Estudiar el efecto del incivismo de forma separada en mujeres y varones contribuye a la necesidad de abordar científicamente las diferencias de género (Ryan \& Branscombe, 2013) y conocer cómo un mismo problema puede afectar a mujeres y varones, pero también qué implicaciones tiene para su bienestar emocional (Garrosa Hernández \& Gálvez Herrer, 2013; López-Zafra \& Garcia-Retamero, 2012; Okimoto \& Heilman, 2012). En definitiva, el presente estudio experimental contribuye al conocimiento del incivismo laboral como fenómeno complejo de relevancia social y en contextos asociados al género.

\section{Agradecimientos}

La investigación ha sido financiada por el Ministerio de Economía y Competitividad. Plan Nacional de $\mathrm{I}+\mathrm{D}+\mathrm{i}$, Subprograma de proyectos de investigación fundamental no orientada Convocatoria 2012. Proyecto FEM2012-34692.

\section{Referencias}

Abad, F. J., Olea, J., Ponsoda, V., \& García, C. (2011). Medición en ciencias sociales y de la salud. Madrid, España: Síntesis.

Agencia Europea para la Seguridad y Salud en el Trabajo. (2006). Las cuestiones de género en relación con la seguridad y la salud en el trabajo. Luxemburgo: Oficina de Publicaciones Oficiales de las Comunidades Europeas.

Andersson, L. M., \& Pearson, C. M. (1999). Tit for tat? The spiraling effect of incivility in the workplace. Academy of Management Review, 24(3), 452-471. doi: 10.2307/259136

Banaji, B. M., \& Hardin, C. (1996). Automatic stereotyping. Psychological Science, 7(3), 136-141. doi: 10.1111/j.14679280.1996.tb00346.x

Banaji, M. R., Hardin, C., \& Rothman, A. J. (1993). Implicit stereotyping in person judgment. Journal of Personality and Social Psychology, 65(2), 272-281. doi: 10.1037/0022-3514.65.2.272

Beattie, L., \& Griffin, B. (2014). Accounting for within-person differences in how people respond to daily incivility at work. Journal of Occupational and Organizational Psychology, 87(3), 625-644. doi: 10.1111/joop.12067

Benokraitis, N. V. (1997). Sex discrimination in the 21st century. In N. V. Benokraitis (Ed.), Subtle sexism: current practice and prospects for change (pp. 5-33). Thousand Oaks, CA: Sage.

Betz, D. E., Ramsey, L. R., \& Sekaquaptewa, D. (2013). Gender stereotype threat among women and girls. In M. Ryan, $\&$ N. Branscombe (Eds.), The SAGE handbook of gender and psychology (pp. 428-450). Londres, Inglaterra: Sage. doi: http://dx.doi.org/10.4135/9781446269930.n26

Bradburn, N. M. (1969). The structure of psychological well-being. Chicago, IL: Aldine.

Chui, C. W. S., \& Dietz, J. (2014). Observing workplace incivility towards women: The roles of target reactions, actor motives, and actor-target relationships. Sex Roles, 71(1-2), 95-108. doi: 10.1007/s11199-014-0367-7

Cortina, L. M. (2008). Unseen injustice: incivility as modern discrimination in organizations. The Academy of Management Review, 33(1), 55-75. doi: 10.2307/20159376

Cortina, L. M., Kabat-Farr, D., Leskinen, E. A., Huerta, M., \& Magley, V. J. (2011). Selective incivility as modern discrimination in organizations: evidence and impact. Journal of Management, 39(6), 1579-1605. doi: $10.1177 / 0149206311418835$

Cortina, L. M., Magley, V. J., Williams, J. H., \& Langhout, R. D. (2001). Incivility in the workplace: incidence and impact. Journal of Occupational Health Psychology, 6(1), 64-80. doi: 10.1037/1076-8998.6.1.64

Diekman, A. B., \& Eagly, A. H. (2000). Stereotypes as dynamic constructs: women and men of the past, present, and future. Personality and Social Psychology Bulletin, 26(10), 1171-1188. doi: 10.1177/0146167200262001

Eagly, A. H. (1987). Sex differences in social behavior: a social-role interpretation. Hillsdale, NJ: Erlbaum. 
Eagly, A. H., Wood, W., \& Diekman, A. B. (2000). Social role theory of sex differences and similarities: a current appraisal. In T. Eckes, \& H. M. Trautmer (Eds.), The developmental social psychology of gender (pp. 123-174). Mahwah, NJ: Erlbaum.

Garrosa Hernández, E., \& Gálvez Herrer, M. (2013). Género y salud laboral. In B. Moreno-Jiménez, \& E. Garrosa Hernández (Eds.), Salud laboral: riesgos laborales psicosociales y bienestar laboral (pp. 67-82). Madrid, España: Pirámide.

Garrosa, E., Carmona-Cobo, I., Moreno-Jiménez, B., \& Sanz-Vergel, A. (2015). El impacto emocional del incivismo laboral y el abuso verbal en el trabajo: el papel protector de la recuperación diaria. Anales de Psicología, 31 (1). doi: http://dx.doi.org/10.6018/analesps.31.1.161494

Heilman, M. E. (1983). Sex bias in work settings: the lack of fit model. In B. Staw, \& L. Cummings (Eds.), Research in organizational behaviour (pp. 269-298). Greenwich, CT: JAI.

Heilman, M. E. (2012). Gender stereotypes and workplace bias. Research in Organizational Behavior, 32, 113-135. doi: 10.1016/j.riob.2012.11.003

Heilman, M. E., \& Eagly, A. H. (2008). Gender stereotypes are alive, well, and busy producing workplace discrimination. Industrial and Organizational Psychology: Perspectives on Science and Practice, 1(4), 393-398. doi: 10.1111/j.17549434.2008.00072.x

Hentschel, T., Heilman, M. E., \& Peus, C. (2013). Have perceptions of women and men changed? Gender stereotypes and self-ratings of men and women. Presentation at the society of personality and social psychology annual conference.

Hershcovis, M. S. (2011). "Incivility, social undermining, bullying... oh my!”: a call to reconcile constructs within workplace aggression research. Journal of Organizational Behavior, 32(3), 499-519. doi: 10.1002/job.689

Hershcovis, M. S., \& Reich, T. C. (2013). Integrating workplace aggression research: Relational, contextual, and method considerations. Journal of Organizational Behavior, 34(S1), S26-S42. doi: 10.1002/job.1886

Instituto Nacional de Seguridad e Higiene en el Trabajo. (2011). VII Encuesta Nacional de Condiciones de Trabajo. Madrid, España: Ministerio de Empleo y Seguridad Social.

Jackson, L. M., Esses, V. M., \& Burris, C. T. (2001). Contemporary sexism and discrimination: the importance of respect for men and women. Personality and Social Psychology Bulletin, 27(1), 48-61. doi: 10.1177/0146167201271005

Kabat-Farr, D., \& Cortina, L. M. (2012). Selective incivility: gender, race, and the discriminatory workplace. In S. Fox, \& T. R. Lituchy (Eds.), Gender and the dysfunctional workplace (pp. 120-134). Northampton, MA: Edward Elgar Publishing.

Kiesler, S. B. (1975). Actuarial prejudice toward women and its implications. Journal of Applied Social Psychology, 5(3), 201-216. doi: 10.1111/j.1559-1816.1975.tb00676.x

Lim, S., Cortina, L. M., \& Magley, V. J. (2008). Personal and workgroup incivility: impact on work and health outcomes. Journal of Applied Psychology, 93(1), 95-107. doi: 10.1037/0021-9010.93.1.95

López-Zafra, E., \& Garcia-Retamero, R. (2012). Do gender stereotypes change? The dynamic of gender stereotypes in Spain. Journal of Gender Studies, 21 (2), 169-183. doi: 10.1080/09589236.2012.661580

López-Zafra, E., Garcia-Retamero, R., \& Eagly, A. H. (2009). Congruencia de rol de género y aspiraciones de las mujeres a posiciones de liderazgo. Revista de Psicología Social, 24(1), 99-108. doi: 10.1174/021347409786923005

Moreno-Jiménez, B., \& Rodríguez-Carvajal, R. (2013). Sociología de la salud laboral. In B. Moreno-Jiménez, \& E. Garrosa Hernández (Eds.), Salud laboral: riesgos laborales psicosociales y bienestar laboral (pp. 43-58). Madrid, España: Pirámide.

Okimoto, T. G., \& Heilman, M. E. (2012). The "bad parent" assumption: how gender stereotypes affect reactions to working mothers. Journal of Social Issues, 68(4), 704-724. doi: 10.1111/j.1540-4560.2012.01772.x

Porath, C., \& Pearson, C. (2010). The cost of bad behavior. Organizational Dynamics, 39(1), 64-71. doi: 10.1016/j. orgdyn.2009.10.006

Ramsey, L. R., Betz, D. E., \& Sekaquaptewa, D. (2013). The effects of an academic environment intervention on science identification among women in STEM. Social Psychology of Education, 16(3), 377-397. doi: 10.1007/ s11218-013-9218-6 
Ryan, M., \& Branscombe, N. (2013). Understanding gender: methods, content, and controversies. In M. Ryan, \& N. Branscombe (Eds.), The SAGE handbook of gender and psychology (pp. 3-11). Londres, Inglaterra: Sage. doi: http://dx.doi.org/10.4135/9781446269930.n1

Schat, A., \& Kelloway, E. K. (2005). Workplace violence. In J. Barling, E. K. Kelloway, \& M. Frone (Eds.), Handbook of workplace stress (pp. 189-218). Thousand Oaks, CA: Sage.

Schein, V. E. (2001). A global look at psychological barriers to women's progress in management. Journal of Social Issues, 57(4), 675-688. doi: 10.1111/0022-4537.00235

Swim, J. K., Mallett, R., \& Stangor, C. (2004). Understanding subtle sexism: detection and use of sexist language. Sex Roles, 51(3-4), 117-128. doi: 10.1023/B:SERS.0000037757.73192.06

Tougas, F., Brown, R., Beaton, A. M., \& St-Pierre, L. (1999). Neosexism among women: the role of personally experienced social mobility attempts. Personality and Social Psychology Bulletin, 25(12), 1487-1497. doi: $10.1177 / 01461672992510005$

Williams, J. E., \& Best, D. L. (1990). Measuring sex stereotypes: a multinational study (revised edition). Beverly Hills, CA: Sage Publications.

Wood, W., \& Eagly, A. (2002). A cross-cultural analysis of the behavior of women and men: implications for the origins of sex differences. Psychological Bulletin, 128(5), 699-727. doi: 10.1037/0033-2909.128.5.699

\section{Endereço para correspondência}

icarmona@uct.cl
Recebido em: 19/03/2014

Revisado em: 11/11/2014

Aprovado em: 01/12/2014 